Elsevier Editorial System(tm) for Scripta Materialia

Manuscript Draft

Manuscript Number: SMM-08-648R1

Title: Creep fracture and load transfer in metal-matrix composite

Article Type: Regular Article

Section/Category:

Keywords: Metal matrix composites (MMCs); Aluminum alloys; Powder processing, Creep rupture.

Corresponding Author: Dr Gaspar González-Doncel, Ph.D.

Corresponding Author's Institution: CENIM, C.S.I.C.

First Author: Ricardo Fernández, Ph.D.

Order of Authors: Ricardo Fernández, Ph.D.; Gaspar González-Doncel, Ph.D.

Manuscript Region of Origin:

Abstract: Creep rupture time data of discontinuously reinforced 6061Al-15vol\%SiCw metal matrix composite, MMC, have been analyzed. Well known phenomenological models, usually applied to high temperature structural metals, such as those described by the Monkman-Grant and Larson-Miller equations, have been used. Consistent results are obtained when data are analyzed in the context of the effective stress borne by the metallic matrix. Such analysis supports further the relevance of a load transfer mechanism during creep of these MMCs, as previously suggested.

Suggested Reviewers: 


\title{
Creep fracture and load transfer in metal-matrix composite
}

\author{
Ricardo Fernández ${ }^{\S}$, Gaspar González-Doncel*
}
Dept. of Physical Metallurgy, Centro Nacional de Investigaciones Metalúrgicas (CENIM), C.S.I.C., Av. de Gregorio del Amo 8, E-28040 Madrid, Spain
$\S$ Present address, Thin Film R\&D Dept. INDO, SA, C/ Alcalde Barnils 72, 08174 Sant Cugat del Vallés, Barcelona, Spain
*Corresponding author, ggd@cenim.csic.es

\begin{abstract}
Creep rupture time data of discontinuously reinforced $6061 \mathrm{Al}-15 \mathrm{vol} \% \mathrm{SiC}_{\mathrm{w}}$ metal matrix composite, MMC, have been analyzed. Well known phenomenological models, usually applied to high temperature structural metals, such as those described by the Monkman-Grant and Larson-Miller equations, have been used. Consistent results are obtained when data are analyzed in the context of the effective stress borne by the metallic matrix. Such analysis supports further the relevance of a load transfer mechanism during creep of these MMCs, as previously suggested.
\end{abstract}

Key words: Metal matrix composites (MMCs); Aluminum alloys; Powder processing, Creep rupture.

In a previous work [1], the creep behavior of a powder metallurgical, PM, 6061Al$15 \mathrm{vol} \% \mathrm{SiC}_{\mathrm{w}}$ composite and the corresponding un-reinforced $6061 \mathrm{Al}$ alloy, also obtained by PM, was investigated. Usually, the creep deformation rate of discontinuously reinforced metal matrix composites, MMCs, has been explained on the basis of the creep behavior of the matrix alloy and assuming the presence of a threshold stress term, $\sigma_{0}$, such that the following powerlaw creep relation is satisfied between the steady state or minimum creep rate, $\dot{\varepsilon}_{\text {min }}$, and the applied stress, $\sigma$,

$$
\dot{\varepsilon}_{\text {min }}=A^{\prime}\left(\frac{\sigma-\sigma_{0}}{E}\right)^{n} \exp \left(-\frac{Q_{c} / R T}{R T}\right)
$$

where $A^{\prime}$ is a material's microstructure constant, $E$ the Young's modulus, $n$ the stress exponent, $Q_{c}$ the activation energy for creep, $R$ the universal gas constant $(R=8.314 \mathrm{~kJ} / \mathrm{mol} \mathrm{K})$, and $T$ the absolute temperature. Attempts to understand the significance of $\sigma_{0}$ on the basis of particle-dislocation interaction micro-mechanisms, however, have failed. On the contrary, it was shown in [1], that composites' creep behavior can be understood in terms of the substructure invariant Sherby's model [2] ( $n=8$ in equation (1)) and replacing $\sigma_{0}$ by the load 
carried by the reinforcement, $\sigma_{T}$. In other words, the composite creep rate is dictated by that of the matrix alloy which sustains only part of the applied stress, $\left(\sigma-\sigma_{T}\right)$, due to a partition effect, (under the hypothesis that no damage or deleterious reactions at the metal-ceramic interface occur). In this manner, speculations about the meaning of $\sigma_{0}$ are avoided and a solid new framework of composite creep deformation at high temperatures can be assumed. The analysis of the experimental results obtained in [1] was assessed by Shear-Lag and Eshelby models of the load carried by the reinforcement. Also, the trends shown from the analysis of data of a number of investigations in the literature on aluminum alloy matrix composites and corresponding un-reinforced alloys confirmed the model proposed in [1].

An important consequence of this new view is that any matrix-dislocation-based plasticity model attempting to account for the creep of the metallic matrix alloy should consider that the actual stress which matrix dislocations bear is $\left(\sigma-\sigma_{T}\right)$. This is contrary to the former view of equation (1) (with $\sigma_{o}$ instead of $\sigma_{T}$ ) in which the stress that the dislocations undergo is directly $\sigma$ [3].

The purpose of the present research is to validate further this new view of composite creep behavior from creep rupture data obtained from the above PM composite (denoted as E219) and the corresponding un-reinforced alloy (denoted as E220). To this objective, available models accounting for phenomenological correlations between time to failure and creep data will be used [4-6]. The development of such models, which extrapolate the data obtained from laboratory creep tests, was promoted by the need to know the creep life of engineering structures designed to operate over time scales that can well exceed decades. Typically, laboratory tests are carried out over much shorter time periods, ranging from some few hours up to several months. Obviously, the final objective of these predictions is to prevent catastrophic creep rupture during component service operation. The predictive capacity of these models is, hence, crucial for a safe service during the time period for which these structures were designed. One of the key features that guaranties this capacity is that the underlying creep/damage mechanisms which dominate materials deformation should be same in the short-term creep tests and in the long-term real service condition. Therefore, appropriate data analysis in light of these models may shed further understanding on the creep deformation of structural materials.

It will be shown here that very consistent results between the time to rupture data from the above 6061 Al-15vol\% $\mathrm{SiCw}$ PM composite and the corresponding un-reinforced alloy are obtained under the assumption that a load partition process during composite creep deformation is operative. 
The materials investigated, the PM processing route, and the experimental procedure for the creep tests are well described elsewhere [1,7]. Briefly, powder of 6061Al alloy of average particle size less than $45 \mathrm{~mm}$ [7] was mixed in a ball-mill with SiC whiskers 20-40 $\mu \mathrm{m}$ in length and average diameter of $0.4 \mu \mathrm{m}$ [8]. The resulting blend was extruded into a cylindrical bar. The same PM route was used to prepare the 6061Al bulk alloy. Tensile creep tests were carried out under constant stress (4-50 $\mathrm{MPa}$ ) provided by an Andrade cam and in the temperature range of $573-723 \mathrm{~K}\left(300-450^{\circ} \mathrm{C}\right)$. Cylindrical creep specimens $10 \mathrm{~mm}$ in the gauge length and $3 \mathrm{~mm}$ in diameter with threaded heads were machined parallel to the extrusion axis. A load cell, inserted in the loading system, allowed monitoring of the applied load. The sample elongation was measured through LVDTs suitably attached to the sample. Test data was stored into a computer through appropriate data acquisition boards. Scanning electron microscopy, SEM, was used to investigate the fracture surface of materials.

The results and the analysis of the minimum creep rate dependence with the applied stress were presented in [1]. It was seen that typical power law dependence with high stress exponents, in the range of 12.3-27.4, were obtained. The analysis of [1] demonstrated that the improved creep response of the composite could be explained on the basis of a load transfer mechanism. Here, the time to failure data will be analyzed within the same framework.

In Figure 1, the time to failure data of the un-reinforced alloy (Figure 1a) and of the composite (Figure 1b) are plotted as a function of the minimum creep rate in a double logarithmic scale (Monkman-Grant plots). Some differences between the un-reinforced alloy and composite data trends can be appreciated. It is seen that the data for the un-reinforced alloy fit quite well in a common line, Figure 1a), in agreement with the Monkman-Grant, MG, equation [4,5],

$$
t_{f} \dot{\varepsilon}_{\min }^{n^{\prime}}=C
$$

where $t_{f}$ is the time for creep rupture and $n^{\prime}$ and $C$ are constants. The constant $C$ is known as the Monkman-Grant constant, and would represent the total elongation to failure in case that $n^{\prime}=1$ and $\dot{\varepsilon}_{\min }$ dominates during the creep test. $n^{\prime}=0.80$ and $C=0.90$ is obtained. The good common fit obtained $\left(\mathrm{R}^{2}=0.98\right)$ at any of the temperatures and stresses investigated is remarkable.

Several trials to derive the $\mathrm{MG}$ equation (with $n^{\prime}=1$ ) from high temperature fracture mechanisms have been attempted, but none of them, however, can be considered as a conclusive theoretical deduction of the equation from microstructural basis (see for example references [9-11]). Although only with an exponent $n^{\prime}=1$ the constant $C$ is sense (strain), the idea underlying this equation (plastic deformation is the macroscopic manifestation of the 
cumulative damage generated during creep) can be also extended to the majority of cases in which $n^{\prime} \neq 1$. Despite the empirical nature of the Monkman-Grant relation, equation 2 , the good fit obtained in figure 1 reveals common inherent deformation/failure mechanisms in this alloy at any of the testing conditions investigated. The time to failure behavior of this alloy in the range of temperatures and applied stresses investigated here is fully predictable from equation 2. At this point it is difficult to envisage an explanation from the deviation of the value of $n^{\prime}$ obtained from the "ideal" $n^{\prime}=1$. A deep analysis of this result is beyond the scope of the present research. It is likely, however, that other processes, such as changes in the microstructure associated with the simultaneous ageing process which occurs during testing, are also important for this result.

For the composite (E219 material) the data, Figure 1b, also obeys the MG relation although the fit is more scattered due to the "rougher" microstructure associated with the presence of the reinforcement. The slope obtained is now $n^{\prime}=0.67$, which separates more from the value of $n^{\prime}=1$ than the data of the un-reinforced alloy. Again, a full explanation of this value is at present, speculative. It may be suggested, however, that the deformation and damage mechanism which operate in the un-reinforced alloy are also involved in the composite. Of course, additional processes linked to the metal ceramic interface may be also operative. These phenomena, however, should be not of great relevance as long as previous work [1] suggests that these mechanisms are minimized. On the other hand, it is possible that the difference between the value of $n$ ' obtained for the un-reinforced alloy and the composite material could rise from the combined action of the load partitioning phenomenon and the increased pipe diffusion (associated with a larger dislocation density in the composite than in the un-reinforced alloy) in the composite material.

It is also seen that the fit for the composite data runs below that for the alloy: For a given value of $\dot{\varepsilon}_{\text {min }}, t_{f}$ for the un-reinforced alloy is almost two orders of magnitude greater than the composite; i.e., fracture occurs earlier in the latter material. This is at a first glance a surprising result which accounts for the effect of the reinforcing ceramic particles in the composite: For a given $\dot{\varepsilon}_{\min }$ the applied stress in the composite is higher than in the alloy. However, this does not undermine the idea that similar inherent deformation and rupture mechanisms would characterize the alloy and the composite. In agreement with Decker, Groza, and Gibeling [12], the correlation of creep rupture data with equation 2 indicates that creep rupture is controlled by the creep deformation mechanisms.

The trends shown in Figure 1, hence, may suggest that common creep deformation mechanisms are active in both the composite and the alloy and that matrix creep behavior 
dominates composite deformation at high temperature. Despite these findings, however, no hint of the load transfer mechanism appears from the plots shown in Figure 1. This is because the applied stress is not a variable to be considered in predictions from the Monkman-Grant relation.

Another phenomenological model for materials creep life prediction, probably the most widely used in engineering, is that described by the Larson-Miller parameter, $P_{L M}$ [6]. This model relates $P_{L M}$ with $\sigma, T$, and $t_{f}$ assuming that equation (2) is obeyed. The model operates, hence, considering the applied stress as an independent factor. The Larson-Miller parameter model is derived from the Arrhenius dependence of the minimum creep rate and considering that equation (2) is obeyed. Accordingly, it is obtained [6]:

$$
P_{L M}=T\left(\log \left(t_{f}+K\right)\right.
$$

Where $K$ is a constant obtained from the experimental data and is equal to $K=46$. For simplicity, the same $K$ value for both the unreinforced alloy and the composite has been assumed given the reasonable similar microstructures and creep deformation mechanisms of both materials [1].

The Larson-Miller parameter varies with stress such that at a given stress level, there are different combinations of T and $t_{f}$ which lead to the same $P_{L M}$ for a given stress.

As it was shown in figure 4 of [1], the power law dependence is obeyed for both the unreinforced alloy and the composite. Considering also the fits shown in figure 1, it is expected that reasonable Larson-Miller plots can be obtained for these materials. This is confirmed by the trends shown in the plot of Figure 2a. In this figure, the Larson-Miller plot of $\sigma$ as a function of $P_{L M}$ is shown for the un-reinforced alloy and the composite data of this investigation. It is seen that distinguishable behaviors for each material are obtained. Both trends run nearly parallel but separated from each other. As suggested above from previous study of these authors [1], however, an appropriate analysis of composite creep data should consider the role of the load transfer mechanisms from matrix to the reinforcement: i.e., that the actual stress carried by the plastically deforming phase of the composite, the metallic matrix, is $\left(\sigma-\sigma_{T}\right)$ instead of the applied stress, $\sigma$. In reference [1] an appropriate separation of the matrix strengthening due to a finer matrix composite microstructure and load transfer strengthening has been made. This allowed calculation of the precise effective stress carried by the composite matrix (and in turn, the load carried by the reinforcement). The data under the different conditions, as calculated in [1] by comparing the creep behavior of the composite and the un-reinforced alloy, are summarized in Table I. Consequently, a plot similar to that 
shown in Figure 2a) but considering the effective stress, $\left(\sigma-\sigma_{T}\right)$, instead of $\sigma$, can be now constructed. As a result from this analysis, the tendency shown in the plot of Figure $2 b$ is obtained. Here, the same data obtained to represent the plot of Figure 2a has been re-plotted, but considering that it is the effective stress, $\left(\sigma-\sigma_{T}\right)$, the one that the composite matrix undergoes during creep deformation. As can be seen here, and contrary to what is appreciated in figure $2 \mathrm{a}$, both the un-reinforced alloy and the composite follow a remarkably common behavior, accounting, hence, for 1) the relevant role played by the load transfer mechanisms during creep and fracture deformation and 2) the common inherent deformation/fracture mechanisms in both the un-reinforced alloy and the composite of this investigation. Despite the fact that the fit in Fig. $2 b$ diverges somewhat with respect to that for the un-reinforced alloy in Fig.2a, the good agreement of all data is remarkable.

Finally, a comparison of the fracture surface of the un-reinforced alloy and the composite is shown from the SEM pictures of Figure 3. The micrograph of Figure 3a) is for the unreinforced alloy and Figure $3 \mathrm{~b}$ ) for the composite. The surfaces correspond to samples in which fracture occurred under similar testing conditions. As can be seen, a typical ductile fracture, with the presence of dimples associated with local plastic deformation in the interior of the grains and, hence, to a trans-granular type of fracture, is apparent. The composite also shows the presence of the reinforcing particles around which the plastically deformed regions are formed. In essence, these micrographs also suggest that similar deformation/fracture mechanisms are underlying in the creep process of these materials.

In summary, it has been shown that time to failure data of aluminum alloy metal matrix composite, as analyzed assuming a load transfer mechanisms and comparing data with that of the corresponding un-reinforced alloy lead to consistent results which validates further the relevant role of load partition during composite creep deformation.

Financial support from MEC (Ministerio de Educación y Ciencia), Spain, under project MAT05-00527 is acknowledge.

[1]- R. Fernández, G. González-Doncel, Acta Mater. 56 (2008) 2549.

[2] O.D. Sherby, R.H. Klundt, A.K. Miller, Metall Trans. 8A (1977) 843.

[3] K-T. Park, E.J. Lavernia, F.A. Mohamed, Acta Metall Mater. 38 (1990) 2149.

[4] F.C. Monkman, N.J. Grant. Proc ASTM. 56 (1956) 593.

[5] D.C. Dunand, B.Q. Han, A.M. Jansen, Metall Mater Trans. A30 (1999) 829. 
[6] F.R. Larson, J. Miller, Am Soc Mech Eng. 74 (1952) 756.

[7] R. Fernández, G. González-Doncel, J Alloys Compd. 440 (2007) 158.

[8] A. Borrego, J. Ibáñez, V. López, M. Lieblich, G. González-Doncel, Scripta Mater. 34 (1996) 471.

[9] G.H. Edwards, M.F. Ashby. Acta Metall. 27. (1979) 1505.

[10] A.Needleman, J.C. Rice, Acta Metall. 28 (1980) 1315.

[11] A.C.F. Kocks, M.F. Ashby. Prog Mater Sci. 27 (1982) 189.

[12] M.W. Decker, J.R. Groza, J.C. Gibeling, Mater Sci Eng. A369 (2004) 101. 


\begin{tabular}{|c|c|c|}
\hline $\begin{array}{c}\text { Temperature } \\
(\mathbf{K})\end{array}$ & $\begin{array}{c}\text { Applied stress } \\
\text { (MPa) }\end{array}$ & $\begin{array}{c}\text { Time to fracture } \\
\text { (s) }\end{array}$ \\
\hline 523 & 40 & $7,2 \mathrm{E}+05$ \\
\hline 523 & 48 & $1,6 \mathrm{E}+05$ \\
\hline 523 & 49 & $9,0 \mathrm{E}+04$ \\
\hline 523 & 49 & $2,4 \mathrm{E}+05$ \\
\hline 523 & 53 & $9,8 \mathrm{E}+03$ \\
\hline 523 & 61 & $5,6 \mathrm{E}+02$ \\
\hline & & \\
\hline 573 & 28 & $2,1 \mathrm{E}+06$ \\
\hline 573 & 31 & $1,5 \mathrm{E}+05$ \\
\hline 573 & 37 & $1,2 \mathrm{E}+04$ \\
\hline 573 & 40 & $2,1 \mathrm{E}+04$ \\
\hline 573 & 42 & $3,0 \mathrm{E}+03$ \\
\hline 573 & 47 & $9,0 \mathrm{E}+02$ \\
\hline & & \\
\hline 623 & 23 & $1,1 \mathrm{E}+05$ \\
\hline 623 & 29 & $1,9 \mathrm{E}+04$ \\
\hline 623 & 29 & $3,8 \mathrm{E}+04$ \\
\hline 623 & 39 & $7,7 \mathrm{E}+02$ \\
\hline
\end{tabular}

\begin{tabular}{|c|c|c|c|}
\hline $\begin{array}{c}\text { Temperature } \\
(\mathbf{K})\end{array}$ & $\begin{array}{c}\text { Applied stress } \\
\text { (MPa) }\end{array}$ & $\begin{array}{c}\text { Effective stress } \\
\text { (MPa) }\end{array}$ & $\begin{array}{c}\text { Time to fracture } \\
\text { (s) }\end{array}$ \\
\hline 623 & 35 & 23 & $2,4 \mathrm{E}+06$ \\
\hline 623 & 35 & 23 & $1,6 \mathrm{E}+04$ \\
\hline 623 & 29 & 25 & $9,3 \mathrm{E}+05$ \\
\hline 623 & 37 & 27 & $6,2 \mathrm{E}+04$ \\
\hline 623 & 50 & 41 & $2,7 \mathrm{E}+02$ \\
\hline & & & \\
\hline 673 & 17 & 16 & $3,7 \mathrm{E}+05$ \\
\hline 673 & 23 & 16 & $2,1 \mathrm{E}+06$ \\
\hline 673 & 23 & 20 & $3,5 \mathrm{E}+05$ \\
\hline 673 & 23 & 20 & $7,6 \mathrm{E}+04$ \\
\hline 673 & 30 & 22 & $3,3 \mathrm{E}+04$ \\
\hline 673 & 35 & 27 & $1,1 \mathrm{E}+03$ \\
\hline 673 & 43 & 31 & $3,7 \mathrm{E}+02$ \\
\hline & & & \\
\hline 723 & 12 & 12 & $1,8 \mathrm{E}+05$ \\
\hline 723 & 15 & 13 & $1,9 \mathrm{E}+05$ \\
\hline 723 & 15 & 14 & $4,2 \mathrm{E}+02$ \\
\hline 723 & 17 & 15 & $6,5 \mathrm{E}+03$ \\
\hline 723 & 20 & 19 & $2,3 \mathrm{E}+03$ \\
\hline 723 & 30 & 22 & $4,2 \mathrm{E}+02$ \\
\hline & & & \\
\hline 773 & 5,0 & $5,0^{*}$ & $1,8 \mathrm{E}+05$ \\
\hline 773 & 6,0 & $6,0^{*}$ & $7,1 \mathrm{E}+04$ \\
\hline 773 & 8,0 & $8,0^{*}$ & $8,5 \mathrm{E}+03$ \\
\hline 773 & 13,0 & $13,0^{*}$ & $1,9 \mathrm{E}+03$ \\
\hline
\end{tabular}

Table I.- Creep conditions and fracture data for the PM A16061 alloy (E220) and A16061$15 \% \mathrm{volSiC}_{\mathrm{w}}$ composite material (E219). 

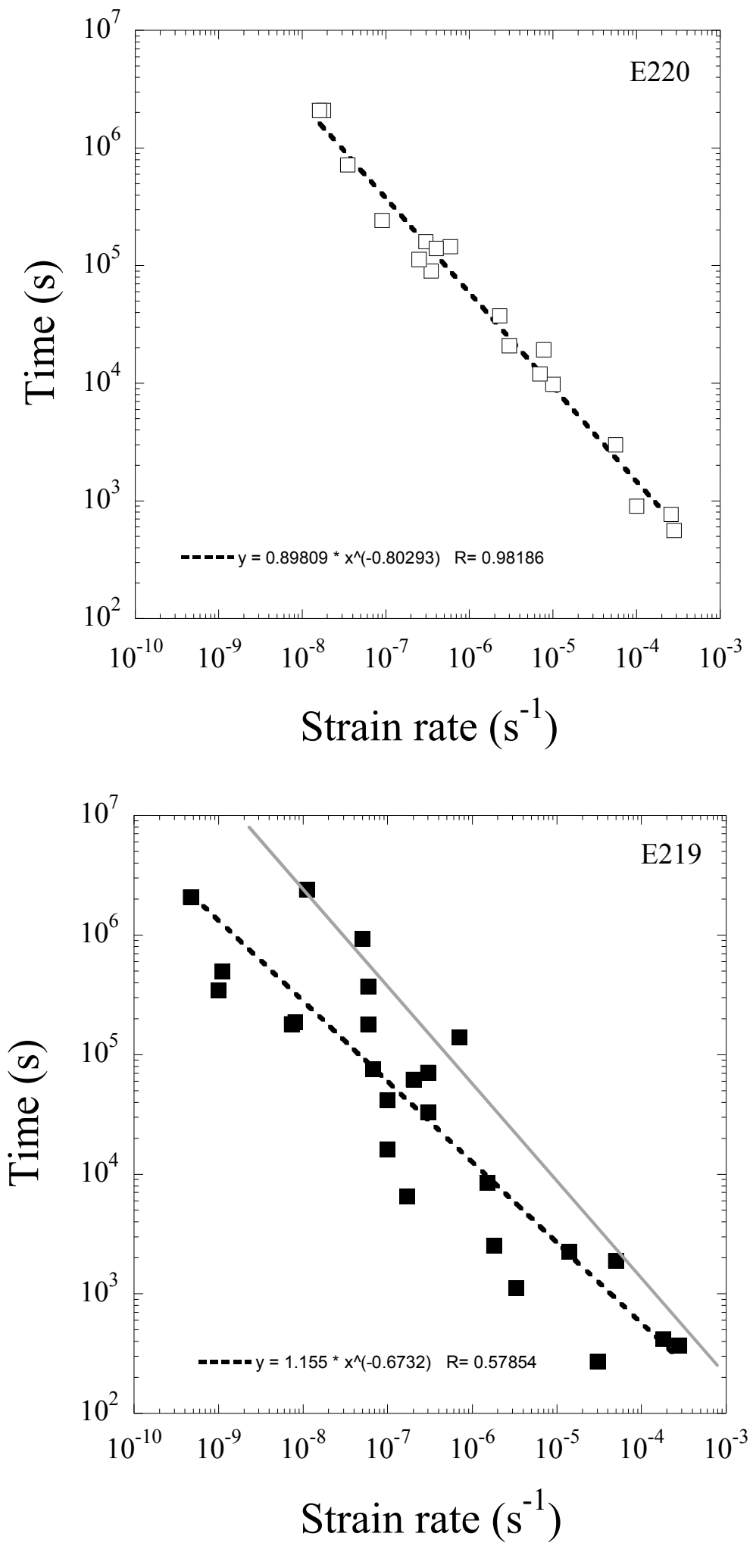

Figure 1.- Time to failure as a function of the minimum strain rate for a) the un-reinforced alloy E220 and b) the composite E219 (Monkman-Grant plots). The data for the un-reinforced alloy fit quite well in a common line for any of the testing conditions investigated. The gray solid line in b) represents, for comparison, the fit obtained for the un-reinforced alloy data. 


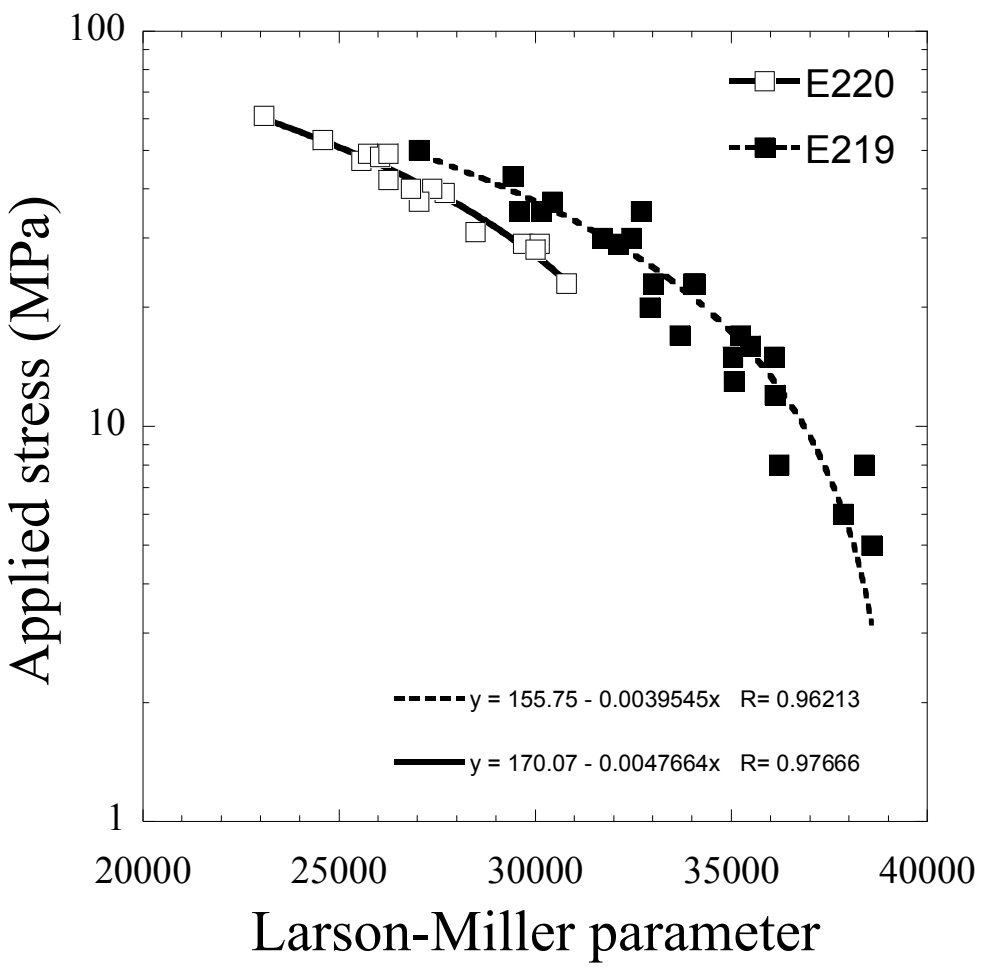

a)

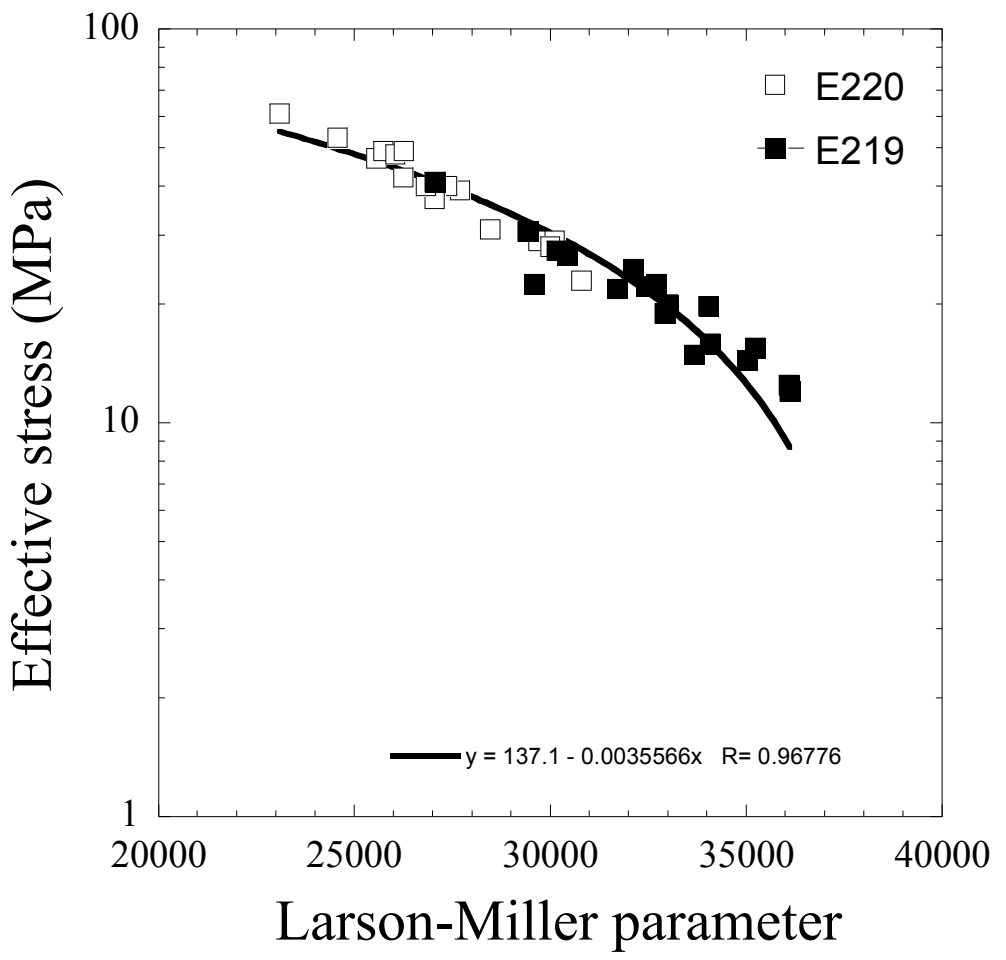

b)

Figure 2.- Larson-Miller plot for the un-reinforced alloy and the composite data. a) The applied stress $v s$. the L-M parameter for both materials. b) The effective stress $\left(\sigma-\sigma_{T}\right)$ on the aluminium matrix alloy of the composite (and the applied stress for the un-reinforced alloy) $v s$. the L-M parameter. By definition, the value of $P_{L M}$, varies with the stress through the empirical constant $K$. The good correlation between data for the un-reinforced alloy and the composite, assuming the effective stress in the latter case, is remarkable. 


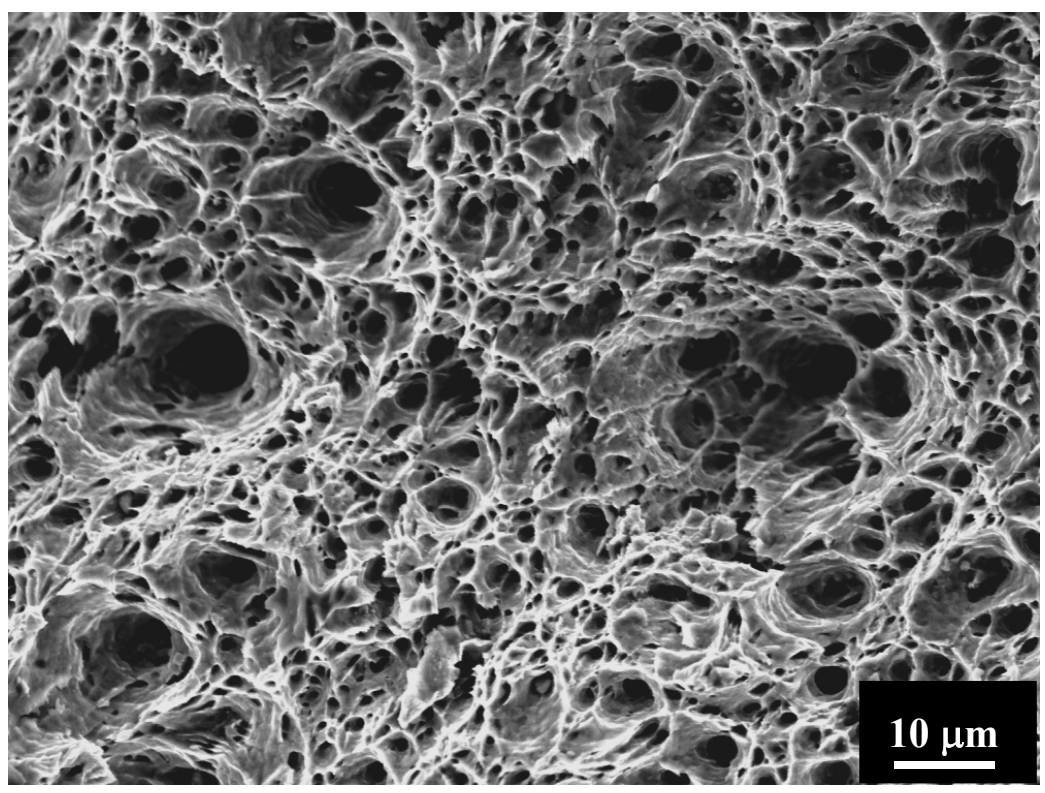

a) $\mathrm{Al} 6061 ; 623 \mathrm{~K} 39 \mathrm{MPa}$.

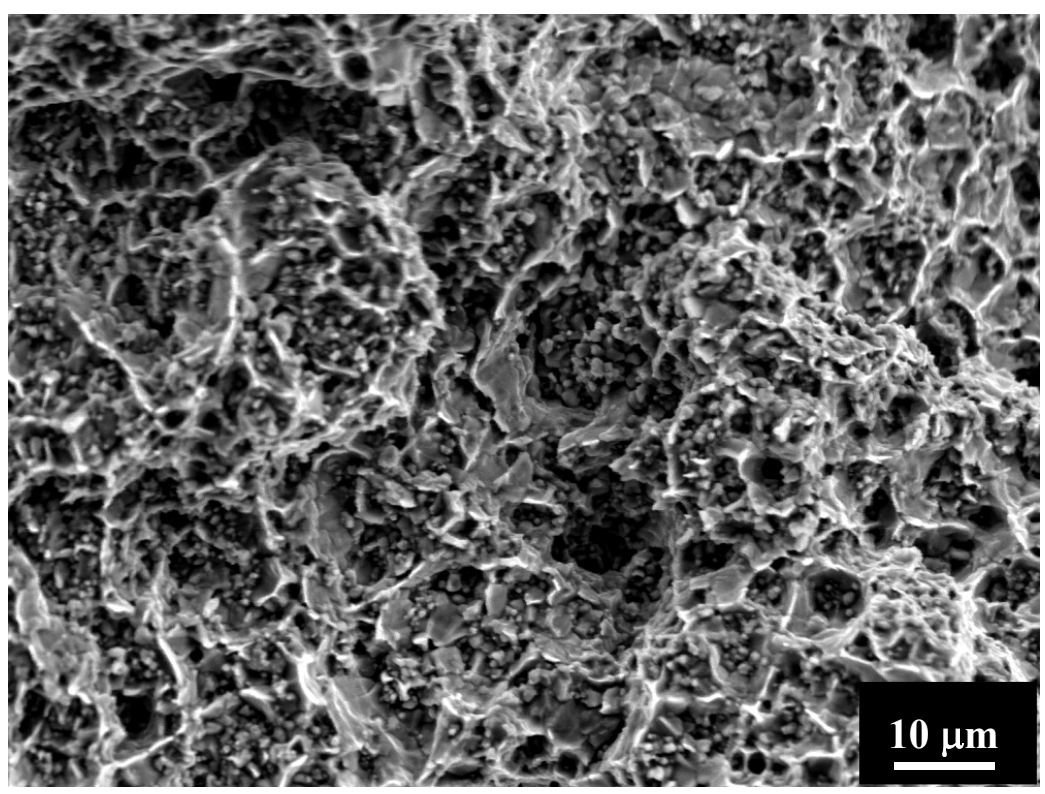

b) $\mathrm{A} 16061+15 \% \mathrm{VolSiC}_{\mathrm{w}}$; $623 \mathrm{~K} 42 \mathrm{MPa}$.

Figure 3. Fracture surfaces of a) the un-reinforced alloy and b) the composite tested at $623 \mathrm{~K}$ and applied stress indicated. For the case of the composite the effective stress on the matrix alloy calculated was $34 \mathrm{MPa}$ (applied stress $42 \mathrm{MPa}$ ). Similar aspect surface with dimples associated with a trans-granular and ductile fracture are evident in both micrographs. 